\title{
Electrical measurement capabilities of the cryogenic dual-mode optical power detector
}

\author{
Marit Ulset Nordsveen", Eivind Bardalen", and Jarle Gran* \\ *Justervesenet, Kjeller, Norway \\ mas@justervesenet.no \\ University of South-Eastern Norway, Borre, Norway
}

\begin{abstract}
The dual-mode optical power detector combines two primary measurement methods into one device - photocurrent and thermal detection. The combination of these two methods can be exploited in a self-calibration procedure, where the internal losses of the photodiode absorber are determined using the thermal mode as a reference. Another advantage of the dual-mode detector, which this work focuses on, is that it provides a direct link between radiometric primary measurement methods and the new SI, by extracting the fundamental constants ratio $e / h c$. This makes the dual-mode detector self-assured, and also serves as a validation of the two primary methods, through a cryogenic highaccuracy comparison on one device. In this work, we present the current electrical measurement capabilities of the dual-mode setup at $40 \mathrm{~K}$ and $60 \mathrm{~K}$, to give an indication of the achievable uncertainty level of the $e / h c$ measurement.
\end{abstract}

Index Terms - International System of Units, Metrology, optical detectors, optical metrology, photodetectors, photothermal effects, semiconductor detectors.

\section{INTRODUCTION}

The EMPIR project chipS.CALe [1] aims to develop a selfcalibrating optical power detector, where thermal detection of incoming radiation is used as a reference to determine the internal losses of the photodiode absorber. The incoming radiation is converted either to a photocurrent, as in a traditional photodiode, or to heat, using electrical substitution to determine the power of the absorbed radiation. The photodiode is used as the absorber in both modes.

In one part of the chipS.CALe project, the dual-mode principle is utilised in a cryogenic detector. Material properties, temperature sensors, thermal stability, packaging to ensure thermal equivalence, control and read-out electronics are being optimised to maximise the signal-to-noise ratio at low temperature, to achieve the lowest possible measurement uncertainty. The aim is to perform a high-accuracy measurement to extract the fundamental constant ratio $e / h c$.

The responsivity $R(\lambda)$ in $\mathrm{A} / \mathrm{W}$ of the photodiode is found directly from measuring the photocurrent $i_{\text {photo }}$ in one mode and thermally measuring the optical power $\Phi_{T}$ in the other mode. The responsivity is given by:

$$
R(\lambda)=\frac{i_{\text {photo }}}{\Phi_{T}}=\frac{e \lambda}{h c}(1-\delta(\lambda))(1-\rho(\lambda))
$$

where $\lambda$ is the wavelength of the absorbed radiation, $\delta(\lambda)$ are the internal losses and $\rho(\lambda)$ is the reflection loss. The internal losses could be determined using the self-calibration method where the thermal mode is used as a reference. However, for the extraction of $e / h c$, we need an external reference for the IQD, to avoid a circular argument. As explained in [2], the IQD can be predicted by computer models with high accuracy. The reflection loss $\rho(\lambda)$ will be the same for both measurement modes, as they both use the same absorber and the same source, and hence the reflection loss will cancel.

The wavelength $\lambda$ can easily be measured using a high accuracy wavelength meter. When each element in (1) has been determined, we can extract the ratio $e / h c$. As these fundamental constants are three of the defining constants of the new SI, the dual-mode detector will provide a direct link between two practical primary radiometric measurement techniques and the SI. This serves not only as a validation of the two primary methods for optical power measurements, but it also exploits the principles of the new SI and strengthens the radiometric position within the SI.

This study is limited to the electrical part of the dual-mode measurement. We exclude optical stability and thermal equivalence, and present the current electrical measurement capabilities of the dual-mode detector and its calculation algorithm.

\section{EXPERIMENT}

\section{A. The Dual-mode Detector}

A commercial silicon photodiode (PD) with size $11 \mathrm{~mm} \times 11$ $\mathrm{mm} \times 0.5 \mathrm{~mm}$ functions as both the optical absorber and electrical heater. In optical heating mode, the power is absorbed in the central part of the active layer of the photodiode, while in electrical heating mode, the power is dissipated near the topside cathode ring by applying a forward bias to the photodiode. Simulations predict that thermal inequivalence of optical and electrical heating is below $1 \mathrm{ppm}$ for the presented structure.

Fig. 1 shows a schematic and image of the dual-mode detector used in this study. A thin $(<200 \mu \mathrm{m})$ PCB with $18 \mu \mathrm{m}$ thick copper tracks is used for electrical connections to the photodiode and temperature sensor via wire bonds and soldered wires. The temperature sensor is bonded with epoxy and wire bonded to the PCB, which is thermally coupled to the photodiode with a thermal diffuser element, consisting of a layered silicon/epoxy structure. The PCB is bonded on a thermally insulating aerogel spacer, which in turn is bonded on 
a machined copper heat sink. Copper wires are soldered to the edge of the PCB, forming the electrical connections to the external circuitry. These wires also form the main thermal link to the heat sink.

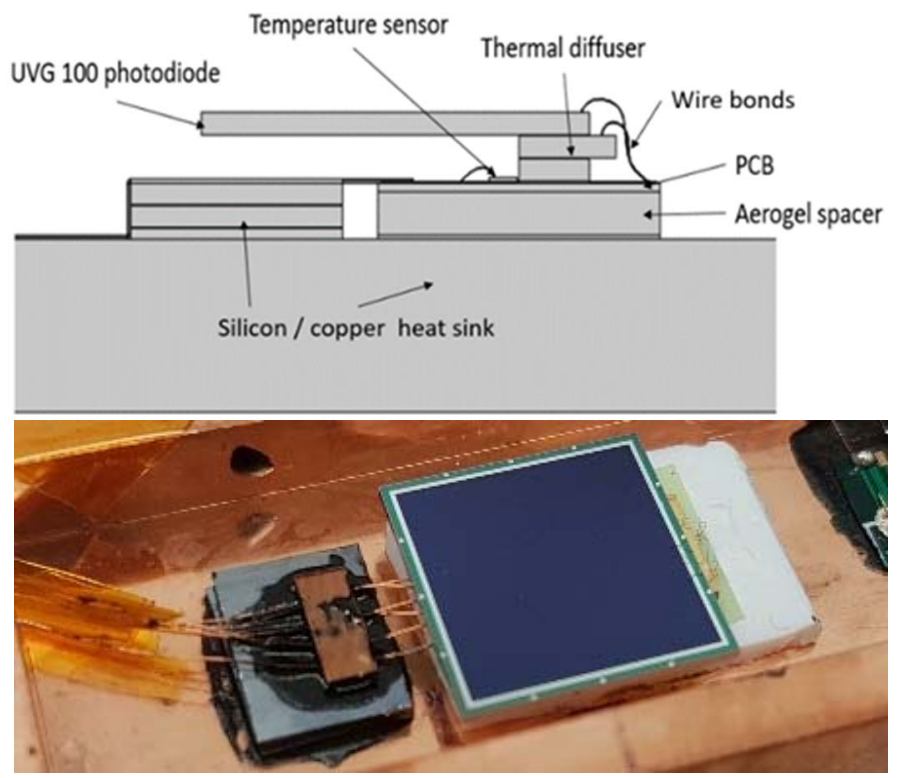

Fig. 1. Schematic (top) and image (bottom) of the cryogenic dual-mode detector.

\section{B. Measurement Setup and Procedure}

In normal operation, the dual-mode detector cycles through both its measurement modes, first converting incoming radiation to a photocurrent, and then to a thermal signal. In thermal mode, the optical power is determined through electrical substitution, where the temperature from optical heating is compared to an equivalent amount of electrical heating. The electrical substitution is carried out by stepping through three heating steps; low electrical heating, optical heating and high electrical heating. The low and high electrical power levels are chosen to be slightly below and above the optical power level. The optical power level is then found from a linear fit, based on the two known electrical power levels, given by $P=I V$.

To verify the calculation algorithms, we replaced the optical heating level with an electrical heating level of known power. The middle electrical heating level was then estimated from the low and high electrical levels, using the calculation algorithms as if the middle level was an optical heating level. The estimated power was compared to the actual power.

During measurements, the detector was screwed on to a second copper heat sink attached to the cold stage of an evacuated cryostat. The temperature signal was measured with a dc Wheatstone bridge, balanced with a mechanically tunable potentiometer. The measurements were performed with 0.5 $\mathrm{mW}$ electrical heating at $40 \mathrm{~K}$ and $60 \mathrm{~K}$ and $5 \mathrm{~mW}$ at $60 \mathrm{~K}$, with a step height of approximately $5 \mu \mathrm{W}$.

\section{RESULTS}

Fig. 2 shows the results for $0.5 \mathrm{~mW}$ at $40 \mathrm{~K}$, while all results are summarised in Table 1. The deviation is larger at $5 \mathrm{~mW}$ than at $0.5 \mathrm{~mW}$. A possible explanation is a larger drift in the temperature signal at $5 \mathrm{~mW}$. The general unfiltered, point-topoint noise level of the temperature reading was $0.6 \mu \mathrm{W}$. In comparison, the noise level of the dc measurement bridge was measured to $0.3 \mu \mathrm{W}$.

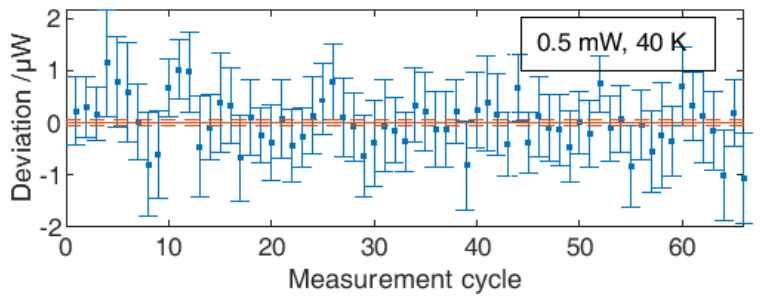

Fig. 2. Relative difference between estimated and measured electrical power for $0.5 \mathrm{~mW}$ at $40 \mathrm{~K}$. Error bars show standard deviation. The red solid and dashed lines show the mean and standard deviation of the mean.

Table 1. Absolute and relative difference between estimated and measured electrical power at $0.5 \mathrm{~mW}$ and $5.0 \mathrm{~mW}$, at $40 \mathrm{~K}$ and $60 \mathrm{~K}$, with type A uncertainty $(k=1)$.

\begin{tabular}{llll}
\hline Power level & $\begin{array}{l}\text { Absolute difference } \\
(\mu \mathrm{W})\end{array}$ & $\begin{array}{l}\text { Relative difference } \\
(\mathrm{ppm})\end{array}$ \\
\hline $\mathbf{4 0} \mathbf{~ K}$ & $0.5 \mathrm{~mW}$ & $-0.0024 \pm 0.060$ & $4.6 \pm 119$ \\
\hline $\mathbf{6 0} \mathbf{~ K}$ & $0.5 \mathrm{~mW}$ & $0.0029 \pm 0.098$ & $5.7 \pm 197$ \\
& $5.0 \mathrm{~mW}$ & $-0.12 \pm 0.06$ & $-24 \pm 12$ \\
\hline
\end{tabular}

\section{CONCLUSION}

The good agreement between the estimated and measured electrical power is promising for a radiometric determination of $e / h c$. Future work will be concentrated around reducing the measurement uncertainty, where the main contribution is the temperature reading. In the future design of the detector, the temperature signal could be increased at the cost of a longer time constant. The signal to noise ratio of the temperature signal could also be improved by replacing the dc bridge with an ac bridge, and this option is being explored.

\section{ACKNOWLEDGEMENT}

This project has received funding from the EMPIR programme co-financed by the Participating States and from the European Union's Horizon 2020 research and innovation programme.

\section{REFERENCES}

[1] J. Gran, Publishable Summary for 18SIB10 chipSCALe. Zenodo. http://doi.org/10.5281/zenodo.3545677, June 2019.

[2] Sildoja, M., et al., "Predictable quantum efficient detector: I. Photodiodes and predicted responsivity." Metrologia vol. 50, no. 4, pp. 385-394, 2013. 